\title{
High Reliability Electricity Pricing Method Research
}

\author{
H.L.WANG ${ }^{1} \&$ W.LONG ${ }^{1}$, R.LIU ${ }^{2}$ \\ ${ }^{1}$ School of Electrical Engineering, Guizhou University, Guiyang,China \\ ${ }^{2}$ State grid Beijing Electric Power Company,Beijing, China
}

KEYWORD: High reliability price; Value engineering theory; Entropy value method; The marginal cost

\begin{abstract}
How to effectively coordinate the reliability and economy in the market environment is becoming a hot spot of today's power market research. Explore viable mechanism of supply and demand based on reliability under market conditions, establish a reasonable reliability service trade mode, the reliability of the market value should be deep mining, make the supply and demand of electricity and reliability can be organically linked. At present, there is little theoretical support for high reliability electricity price. In this paper, some methods in the high reliability electrical price were introduced, and the basic principal, application, advantages and disadvantages were summarized.
\end{abstract}

\section{INTRODUCTION}

Reasonable electricity price should take into account two aspects: on the supply side to reflect the cost of production, the demand side can reflect the load characteristics. The current electricity pricing system only reflect the cost of the supply side and regard power products as the equivalent goods, without taking into account the characteristics of electricity users. In the power market, the price development should follow the economic principles of high quality and high price, it's obvious that the high quality goods more expensive than the low quality of similar goods, which is a basic principle underlying the market economy [1]. Therefore, the character should be reflected that different users have different power supply reliability requirements, but the existing tariff system does not reflect this.

High reliability electricity price refers to different user requirements electricity suppliers provide different levels of power reliability and quality with different prices. At the same time, when the user selects a certain level of supply reliability has not been reached after, the power supplier shall indemnify the user according to the principles of market economy [2].

\section{HIGH RELIABILITY POWER PRICE MODEL BASED ON VALUE ENGINEERING THEORY}

This model based on Value Engineering Theory, the price functional elasticity coefficient $E_{p f}$ has been reflected the sensitivity of price $P$ to function $F$ [3]. When the function is changed from $F_{0}$ to $F_{0}+\Delta F$, the price goes from $P_{0}$ to $P_{0}+\Delta P$, then the function and the relative change in prices as follows:

$E_{p f}=\frac{\Delta P / P_{0}}{\Delta F / F_{0}}$ 
Based on the characteristic of price functional elasticity coefficient $E_{p f}$, when $E_{p f}$ constant equal to 1 and function adjustment orientation has been determined, then the interests of both enterprises and consumers can be taken care, reflecting the principle of fairness. Therefore, the above equation becomes:

$\Delta P=\frac{\Delta F}{F_{0}} P_{0}$

Typically, the power supply reliability index of each load point $i$ in power distribution system are as follow [4]: load loss rate $\lambda_{i}$ (times / year), average interruption duration $r_{i}$ (hours / time), annual average outage time $U_{i}$ (hours / year). In this paper, the load loss rate $\lambda_{i}$ as an example, the high reliability electricity pricing method based on the load loss rate $\lambda_{i}$ will be introduced.

a. The power supply reliability index $\lambda_{i}$ can be calculated through distribution system reliability assessment techniques;

b. In the load $L_{\bar{i}}$ of each load point $i$ as the weighted coefficient to calculate the average load loss rate $\lambda_{0}$

$\lambda_{0}=\sum_{i=1}^{m} \lambda_{i} \times\left(\frac{L_{i}}{L}\right)$

Where $m$ is the number of system load points, $L$ is the total system load, $L_{i}$ is the load of each load point $i$.

c. Assuming the original base price is $P_{0}$ (yuan $/(\mathrm{kW} \bullet \mathrm{h})$ ) corresponds to average loss load rate $\lambda_{0}$ in this system, the change of reliability electricity price $P_{i}\left(\lambda_{i}\right)$ at each load point $i$ could been calculated through equation (2) and (3).

The high reliability electricity price $P_{i}\left(\lambda_{i}\right)$ of each load point $i$ as follows:

$$
\left|\Delta P_{i}\right|=\frac{\left|\Delta \lambda_{i}\right|}{\lambda_{0}} P_{0}=\frac{\left|\lambda_{i}-\lambda_{0}\right|}{\lambda_{0}} P_{0}
$$


$P_{i}\left(\lambda_{i}\right)\left\{\begin{array}{ll}P_{0}+\left|\Delta P_{i}\right|=\left(2-\frac{\lambda_{i}}{\lambda_{0}}\right) P_{0} & \lambda_{i}<\lambda_{0} \\ P_{0} & \lambda_{i}=\lambda_{0} \\ P_{0}-\left|\Delta P_{i}\right|=\left(2-\frac{\lambda_{i}}{\lambda_{0}}\right) P_{0} & \lambda_{0}<\lambda_{i} \leq \delta \lambda_{0} \\ P_{0 \text { min }} & \lambda_{i}>\delta \lambda_{0}\end{array} \quad\right.$ Wherein, $1<\delta<2 。$

From equation (5) can be seen, when $\lambda_{i} \leqslant \lambda_{0}, P_{i}\left(\lambda_{i}\right)>P_{0}$; when $\lambda_{i}=\lambda_{0}, P_{i}\left(\lambda_{i}\right)=P_{0}$; when $\lambda_{i}>\lambda_{0}, P_{i}\left(\lambda_{i}\right)<P_{0}$. The high reliability pricing method is effective reflects the principal of "High quality high price, poor quality low price." In order to avoid speculation of individual users, protection basic earnings of power suppliers, when $\lambda_{i}>\delta \lambda_{0}$, the lowest system reliability electricity price is $P_{0 \text { min }}$, the specific value could been determined by the supplier according to their operating costs.

\section{JOINT PRICING MODEL BASED ON MULTI-INDEX ENTROPY METHOD}

Through value engineering theory approach, we can get different high reliability electricity price about all the reliability index. There are some interrelated relationships between different reliability index, so a single reliability index does not fully reflect the reliability of the electricity properties. After a comprehensive calculated to all reliability electricity prices can we get a joint pricing model based on multi-index Entropy Method.

This paper introduce a joint pricing model based on multi-index Entropy Method according to three reliability indexs: load loss rate $\lambda_{i}$ (times / year), average interruption duration $r_{i}$ (hours / time), annual average outage time $U_{i}$ (hours / year).

$P_{i}=\alpha_{i}\left(\lambda_{i}\right) P_{i}\left(\lambda_{i}\right)+\beta_{i}\left(r_{i}\right) P_{i}\left(r_{i}\right)+\gamma_{i}\left(U_{i}\right) P_{i}\left(U_{i}\right)$

$\alpha_{i}, \beta_{i}$ and $\gamma_{i}$ are the weights of different reliability index in the above formula, them reflect the influence of different reliability index to reliability electricity price, the greater weight, the higher influence. Reliability index weights in different load points should satisfy the following formula:

$\alpha_{i}\left(\lambda_{i}\right)+\beta_{i}\left(\gamma_{i}\right)+\gamma_{i}\left(U_{i}\right)=1$

Entropy method is an objective weighting method, it determine the index weight based on the amount of information included in the index value. The larger amount of information, the smaller uncertainty of the system, entropy is also smaller; on the contrary, the smaller the amount of information, the greater uncertainty of the system, the entropy is greater [5].Assuming that there are $m$ load point, index value $x_{i j}(1 \leq i \leq \mathrm{m}, 1 \leq j \leq 3)$.

a. After standardization of various index can we get a standardized matrix $X=\left\{x_{i j}\right\}_{\mathrm{m} \times 3}$, then the weight of $j$ index at $i$ load point can been calculated as the follow formula. 
$Y_{i j}=x_{i j} / \sum_{i=1}^{n} x_{i j}$

b. Calculate the index information entropy:

$e_{j}=-k \sum_{i=1}^{m}\left(Y_{i j} \times \ln Y_{i j}\right)$

Calculate information entropy redundancy:

$d_{j}=1-e_{j}$

d. Calculate the index weight:

$w_{i}=d_{j} / \sum_{j=1}^{3} d_{j}$

Application of the method described above can get all the index weight of reliability electricity price, then the value of $P_{i}$ can been determined.

Joint pricing model based on multi-index Entropy Method to some extent reflects the different needs of different load points for different reliability index. It can be better to reconcile the relationship between various indexs at a certain reliability level and reduce electricity price volatility. Entropy method can objectively determine the weight, reduce man-made certain subjectivity. This method could solve this question that the user is not intuitive feelings for reliability when electricity pricing, which further illustrates the implementation rationality to carry out a partial class pricing for the user.

\section{HIGH RELIABILITY AND MARGINAL COST PRICING MODEL BASED ON THE THEORY OF MARKOV PROCESSES}

The connection mode of power system is usually more complex, So the reliability computation is more complicated. Due to the different power supply reliability under different power supply mode, so the high reliability electricity price should start to study depending on supply reliability rate.

The idea is to divide high reliability price into two parts: the spare circuit royalties and spare circuit standby costs (opportunity costs), calculation without the specific connection mode of loop and specificity of standby power supply [6]. The marginal capacity cost of different voltage levels consist of marginal capacity cost of transmission and distribution $\left(X_{t}\right)$ and marginal capacity cost of electricity generation $(Y)$, making the share proportion of marginal capacity cost as the electricity tariff recovery $\alpha$ and basic price recovery $\beta$ respectively, then:

a. Transmission and distribution capacity price of each voltage level, that is, the recovery part of the basic price .According to proportion between the voltage level of marginal transmission and distribution capacity and cost accounting for the voltage level of the marginal capacity cost, we can calculate the ratio between the voltage level of transmission distribution capacity and price for the voltage level of the basic price. 


$$
P_{t}=\frac{X_{t}}{\left(X_{t}+Y\right) \beta} \times O B_{t}
$$

Where $P_{t}$ is the electricity price of transmission and distribution capacity at different voltage level; $X_{t} /\left(X_{t}+Y\right)$ is the ration that the marginal cost of transmission and distribution capacity at different voltage level account for the total cost of the marginal capacity; $\beta$ is the basic electricity price ratio inside the marginal cost of the total capacity for each voltage level; $X_{t} /\left[\left(X_{t}+Y\right) \beta\right]$ is the basic electricity price recovery ratio inside the marginal cost of power transmission and distribution capacity for each voltage level; $O B_{t}$ is the basic price at different voltage level, yuan (kW / Year).

b. The spare circuit royalties: charging for a certain proportion of transmission and distribution capacity electricity price.

$$
P_{u t}=T \times P_{t}=T \times \frac{X_{t}}{\left(X_{t}+Y\right) \beta} \times O B_{t}
$$

Where $P_{u t}$ is the spare circuit royalties at different voltage level; $T$ is the ratio of spare circuit has been used, $\%$.

c. Reserve price of the standby circuit: the capacity cost of the electricity price recovery. Because the system to provide the user with the reserve capacity, Therefore we should also calculate the user need to pay the standby fee ,that is, he extra opportunity cost, namely the marginal capacity cost recovery from electricity price. According to the ratio between the different voltage level capacity cost marginal transmission and distribution capacity cost and cost of power generation capacity, the ratio relationship between basic electricity price and electricity price, and the proportion from basic price recovery capacity cost, the standby fee of added loop is:

$$
P_{r \tau}=\frac{O B_{\tau}}{\beta} \times \alpha \times u=\frac{\alpha}{\beta} \times u \times O B_{t}
$$

Where $u$ is the utility coefficient of spare circuit.

This part is to make up for a primary circuit is not used and the cost of capacity should be recycled by the electricity degree electricity price, as well to reduce the risks faced by the power supply enterprise computing part charged.

d. The high reliability electricity price of new spare circuit:

$$
\begin{aligned}
& P_{i t}=P_{u t i} \times \rho_{i}+P_{r t i} \\
& =\left[\rho_{i} \times \frac{\alpha}{\beta} \times u_{i}+T_{i} \times \frac{X_{t}}{\left(X_{t}+Y\right) \beta}\right] \times O B_{t}
\end{aligned}
$$


Where: $i=2,3, \ldots n$ (2 represents the second circuit, 3 represents the third circuit, and so on); $P_{i t}$ is the high reliability electricity price of new spare circuit at different voltage level; $\rho_{i}$ is the unused probability of new spare circuit (\%);

$u_{i}$ is the utility coefficient of new spare circuit $\left(u_{1}=1, u_{2}=0.4\right)$.

e. The high reliability electricity price of new spare circuit accounts for the proportion of basic electricity price:

$\mu_{i t}=\frac{P_{i t}}{O B_{t}}=\left(\rho_{i} \times \frac{\alpha}{\beta} \times u_{i}+T_{i} \times \frac{X_{t}}{\left(X_{t}+Y\right) \beta}\right)$

Where $\mu_{i t}$ is the ratio that high reliability electricity price of new spare circuit accounts for the basic electricity price, $\%$.

The method set out to study the high reliability electricity price that should be charged from the users, which is more operational.

\section{CONCLUSION}

Above three kinds of high reliability pricing methods are combine reliability and economy effectively, which the theory of value engineering is the foundation of high reliability price. Not only can it balance the interests of businesses and consumers effectively, but also can reflect the principle of fairness. The multi index comprehensive price which based on entropy method can define the relationship between a given reliability level of each index in objectively, reducing artificial subjectivity, giving more reasonable price. Pricing method based on marginal cost and the theory of Markov processes is used to study the reliability price charged to the user on the basis of power supply reliability rate and without concerned about the specific connection mode of the loop and specificity of the standby power supply, make the concrete implementation of high reliability price is easier to operate.

\section{REFERENCE}

[1] Wu Zhenqiu,Ye Shishun,Kuang Wenkai.Reliability Transaction and Reliability Pricing in Electricity Market Environment[J].Power System Technology,2006,30(4):74-77.

[2] Fan Yuefan.Electricity Sales Price Theory and Empirical Research Based on DSM Project Management[D]. North China electric power university,2007.

[3] Zhou Ping.Reliability and Economy Research on Electricity Market Operation[D].Chongqing University,2004.

[4] Yang Haixia,Xie Kaigui,Cao Kan.Reliability electricity price model for power market considering indicator weight[J].Power System Protection and Control,2011,39(16):67-73.

[5] Deng Yue,Tian Li,Gao Laixing.A power market reliability price model based on the method of entropy[J].Journal of Guizhou Normal University(Natural Sciences),2012,30(5):80-83. 
[6] Wang Wenshan,Wang He,Han Yinghao.Pricing of electricity with high reliability in electricity markets[J].East China Electric Power,2007,35(11):72-75. 